Signal \& Image Processing : An International Journal(SIPIJ) Vol.1, No.2, December 2010

\title{
FACE RECOGNITION USING SIMPLIFIED FUZZY ARTMAP
}

\author{
Antu Annam Thomas ${ }^{1}$ and M. Wilscy ${ }^{2}$ \\ ${ }^{1}$ Saintgits College of Engineering, Pathamuttom, Kottayam, Kerala \\ antuannamegmail.com \\ ${ }^{2}$ Department of Computer Science, University of Kerala, Karyavattom Campus, \\ Trivandrum, Kerala \\ wilsyphilipose@gmail.com
}

\begin{abstract}
Face recognition has become one of the most active research areas of pattern recognition since the early 1990s. This project thesis proposes a novel face recognition method based on Simplified Fuzzy ARTMAP (SFAM). For extracting features to be used for classification, combination of Principal Component Analysis (PCA) and Linear Discriminant Analysis (LDA) is used. This is for improving the capability of LDA and PCA when used alone.PCA reduces the dimensionality of input face images while LDA extracts the features that help the classifier to classify the input face images. The classifier employed was SFAM. Experiment is conducted on ORL, Yale and Indian Face Database and results demonstrate SFAM's efficiency as a recognizer. The training time of SFAM is negligible. SFAM has the added advantage that the network is adaptive, that is, during testing phase if the network comes across a new face that it is not trained for; the network identifies this to be a new face and also learns this new face. Thus SFAM can be used in applications where database needs to be updated frequently. SFAM thus proves itself to be an efficient recognizer when a speedy, accurate and adaptive Face Recognition System is required.
\end{abstract}

\section{KEYWORDS}

Face recognition, Principal Component Analysis, Linear Discriminant Analysis, Neural Network, Simplified Fuzzy ARTMAP.

\section{INTRODUCTION}

Face recognition [1] is an important and a challenging research problem spanning numerous fields and disciplines. Face recognition is attention seeking because, in addition to having numerous practical applications such as bankcard identification, access control, security monitoring, and surveillance system, is a fundamental human behaviour that is essential for effective communications and interactions among people.

Two categories of methods can be employed for face recognition one is global approach or appearance-based approach and second one is feature-based or component-based approach. Among these two categories of solutions to the problem [2] the most successful seems to be appearance-based approaches [3], which generally operate directly on images or appearances of face objects and process the image as two dimensional patterns. These methods extract features to optimally represent faces belonging to a class and to separate faces from different classes. Ideally, it is desirable to use only features having high separability power while ignoring the rest [3].Most of the effort have been used to develop powerful methods for feature extraction [4]-[8] and to employ classifiers like Support Vector Machine (SVM) [9], Hidden Markov Models (HMMs) [10], Neural Networks [11]-[15] for efficient classification.

The main trend in feature extraction has been representing the data in a lower dimensional space. Principal Component Analysis (PCA) [16],[17], [5]-[6] and Linear Discriminant analysis (LDA) [7] are two main techniques used for data reduction and feature extraction in the appearance-based approaches. PCA maximizes the total scatter while LDA maximizes the

DOI : $10.5121 /$ sipij.2010.1212 
Signal \& Image Processing : An International Journal(SIPIJ) Vol.1, No.2, December 2010

between class scatter and minimizes the within class scatter. Thus extraction of features by PCA is such that it is good for dimensionality reduction and data representation tasks while extraction of features by LDA is such that it is good for recognition tasks [18]. But when dimensionality of face images is high, LDA is not applicable and therefore LDA is deprived from its advantage to find effective features for class separability. To resolve this problem PCA and LDA methods are combined [3], PCA is applied to preprocessed face images to get low dimensionality images which are ready for applying LDA.

Finally to decrease the error rate, instead of Euclidean distance criteria which was used in [19], we implement a neural network, specifically SFAM, to classify face images based on its computed LDA features

Earlier many of the face recognition systems used Back Propagation Neural Network (BPNN) as the classifier. But BPNN suffers from Stability Plasticity Dilemma and takes a lot of computer time to converge during the time of training [28]. Not only does SFAM overcomes both these disadvantages but also turns the face recognition system to be adaptive.

The system was tested on ORL database and the test results demonstrated the efficiency of SFAM as a recognizer with high recognition rate and negligible training time. Test results also proved this novel system based on SFAM to be adaptive.

\section{FACE ReCognition Problem}

In the literatures, face recognition problem can be formulated as: given an input face image identify or verify the person in the image. When comparing person verification to face recognition, there are several aspects which differ.

First, during verification, a client - an authorized user of a personal identification system makes an identity claim. Computationally this means that it is not necessary to consult the complete set of database images in order to verify a claim. An incoming image (referred to as a probe image) is thus compared to a small number of model images of the person whose identity is claimed and not, as in the recognition scenario, with every image (or some descriptor of an image) in a potentially large database. Second, an automatic authentication system must operate in near-real time to be acceptable to users. Finally, in recognition experiments, only images of people from the training database are presented to the system, whereas the case of an imposter (most likely a previously unseen person) is of at most importance for authentication.

Face recognition is a biometric approach that employs automated methods to verify or recognize the identity of a living person based on his/her physiological characteristics. In general, a biometric identification system makes use of either physiological characteristics (such as a fingerprint, iris pattern, or face) or behaviour patterns (such as hand-writing, voice, or keystroke pattern) to identify a person. Because of human inherent protectiveness of his/her eyes, some people are reluctant to use eye identification systems. Face recognition has the benefit of being a passive, non intrusive system to verify personal identity in a "natural" and friendly way.

All face recognition algorithms consist of two major parts: (a) face detection and normalization and (b) face identification. Algorithms that consist of both parts are referred to as fully automatic algorithms and those that consist of only the second part are called partially automatic algorithms. This paper presents a partially automatic face recognition system.

\section{FACE RECOGNition Process}

A successful face recognition system should have a feature extractor that is be able to extract features that help the classifier to efficiently classify the input face images. Feature Extraction 
Signal \& Image Processing : An International Journal(SIPIJ) Vol.1, No.2, December 2010

process is thus as important as classification process in every Face Recognition Algorithms. Large amount of effort in object recognition field is thus put into developing methods by which features can be extracted so that the objects are optimally represented and objects of different class are sufficiently separated. Principal Component Analysis (PCA) and Linear Discriminant Analysis (LDA) are two popular tools used for dimensionality reduction and feature extraction in most of pattern recognition applications.

Principal component analysis or karhunen-loeve transformation [20] is standard technique used in statistical pattern recognition and signal processing for data reduction and Feature extraction [21]. As the pattern often contains redundant information, mapping it to a feature vector can get rid of this redundancy and yet preserve most of the intrinsic information content of the pattern. These extracted features have great role in distinguishing input patterns.

Thus PCA extracts features that ensure compact way of representation. But the problem is that PCA not only maximizes the inter class scatter but also the intra class scatter.

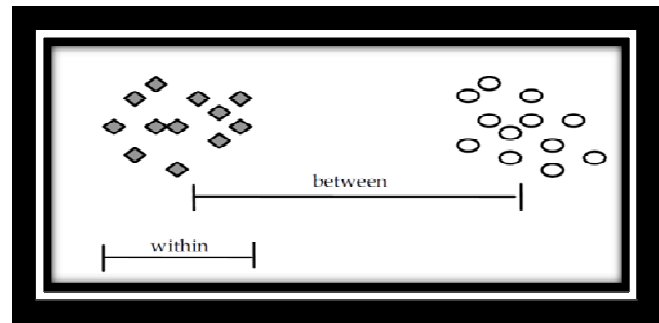

Figure 1. Between-class (Inter-class) and Within-class (Intra-class) scatter

Figure 1 shows the Inter- class and Intra-class scatter. The within class scatter represents how face images are distributed closely within classes and between class scatter describes how classes are separated from each other. Thus to effectively recognize faces features should be extracted so that Inter-class scatter should be maximized and Intra-class scatter should be minimized. But in case of PCA it maximizes both Inter-class and Intra-class scatter.

So we go for a feature extractor that extracts features such that Inter-class scatter is maximized and Intra-class scatter is minimized. LDA is such a feature extractor where Inter-class scatter is maximized and Intra-class scatter is minimized. Thus LDA seeks directions that are efficient for discrimination.

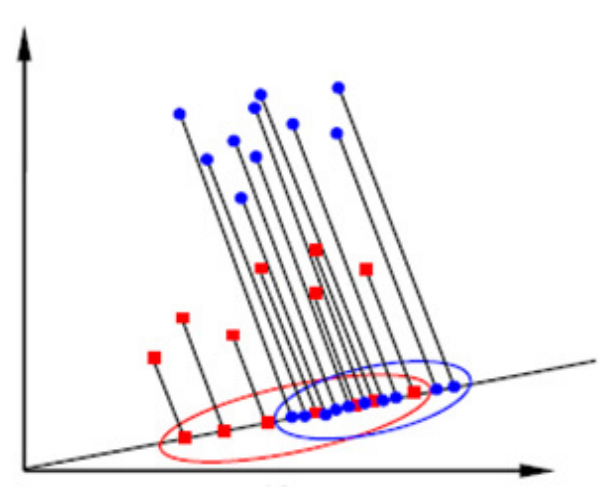

Figure 2(a). Projection of sample points onto a Eigenface space

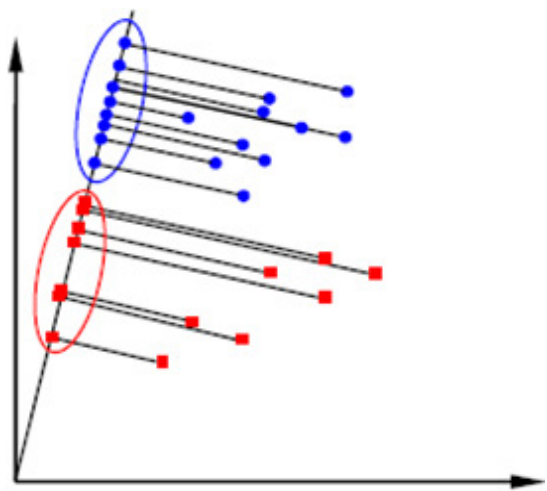

Figure 2(b). Projection of sample points onto a Fisherface space

Figure 2(a) shows PCA (Eigen-faces) maximizing projected total scatter and Figure 2(b) shows LDA (Fisher-faces) maximizing the projected interclass scatter. 
Signal \& Image Processing : An International Journal(SIPIJ) Vol.1, No.2, December 2010

Thus from this discussion it is clear that PCA is of great advantage when dimensionality reduced optimal representation is required but it does not perform better than LDA in recognition tasks [17],[22]. LDA is very efficient in selecting features that are of at most importance in recognition tasks because LDA aims at increasing the inter-class scatter and decreasing the intra-class scatter.

But LDA is disadvantageous when the dimensionality of face images is high. In order to resolve this problem PCA and LDA is combined together so that PCA reduces the dimensionality of the face images before LDA extracts features for recognition purpose.

The process of face recognition thus consists of four main steps Preprocessing, Dimensionality Reduction, Feature Extraction and Classification.

The aim of the face preprocessing step is to normalize the face images, so that a robust feature extraction can be achieved.

The aim of dimensionality reduction is to reduce the dimensionality of the preprocessed face images. PCA is used in this step for dimensionality reduction.

The Feature Extraction step extracts features with high class separability. LDA is used in this step for feature extraction.

The aim of Classification step is to classify the input face image to the correct class to which it belongs.

Euclidean Distance approach is generally used for classification. But this method is error prone. Thus we go for Neural networks which, with its massive parallelism in structure and high computation rates, provide a great alternative to other conventional classifiers and decision making systems.

Back Propagation Neural Network (BPNN) is generally employed as the classifier in Face Recognition tasks. But BPNN suffers from Stability Plasticity Dilemma and High Computer time for training. Thus in this paper a novel approach based on Simplified Fuzzy ARTMAP is investigated.

Simplified Fuzzy ARTMAP overcomes all these disadvantages of BPNN. SFAM transformed the system into an adaptive, speedy and more secure system.

\section{SFAM THE CLASSIFIER}

Simplified Fuzzy ARTMAP is the structure developed by Kasuba in the year 1993. It is a vast simplification of Carpenter and Grossberg's fuzzy ARTMAP. This has a reduced computational overhead and architectural redundancy when compared to its predecessor fuzzy ARTMAP.

SFAM comprises of two layers an input and an output layer (see Figure 3.) [23]-[26]. The input to the network flows through the complement coder. Here the input string is stretched to double the size by adding its complement also. The complement coded input then flows into the input layer and remains there. Weights (W) from each of the output category nodes flows down to the input layer. The category layer just holds the names of the $M$ number of categories that the network has to learn. The match tracking and vigilance parameter of the network architecture are primarily for network training.

$\rho$, the vigilance parameter ranges from 0 to 1 and it controls the granularity of the output node encoding. Thus, high vigilance values make the output node much fussier during pattern encoding and low vigilance allows relaxed matching criteria for the output node. 
Signal \& Image Processing : An International Journal(SIPIJ) Vol.1, No.2, December 2010

The "match tracking" allows the network to adjust its vigilance during learning from some base level, in response to errors in classification during the training phase. It is through match tracking that the network adjusts its own learning parameter to decide when to create new output nodes or reshape its decision regions. During training, match tracking is evoked when the selected output node does not represent the same output category corresponding to the input vectors given [19].

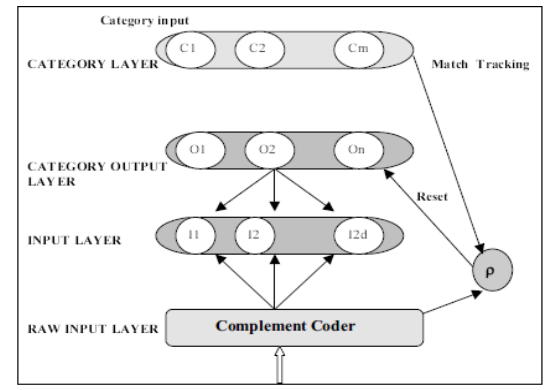

Figure 3. Architecture of SFAM network

Once SFAM has been trained, the equivalent of a "feed forward" pass for an unknown pattern classification consists of passing the input pattern through the complement coder and into the input layer. The output node activation function is evaluated and the winner is the one with the highest value. The category of the input pattern is the one with which the winning output node is associated [23]-[26].

\section{The Proposed System}

Figure 4 shows the proposed recognition system using SFAM as the classifier. Figure 4(a) shows the training phase and Figure 4(b) shows the testing phase.

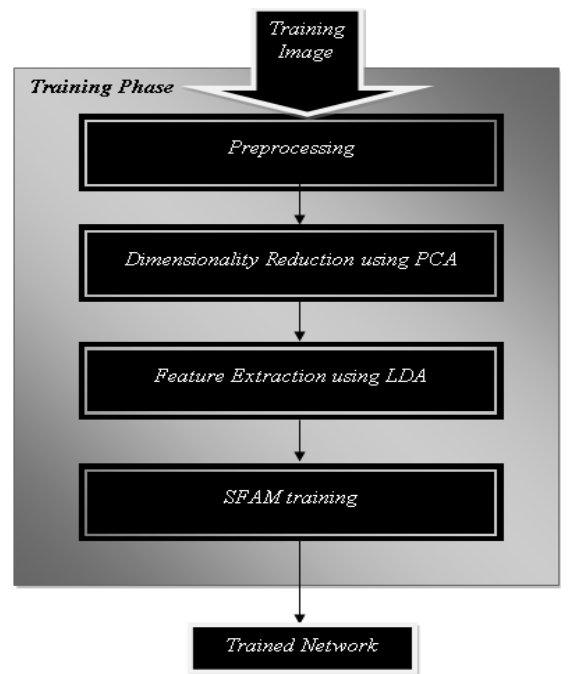

Figure 4(a). Training Phase of the Proposed Face Recognition System 


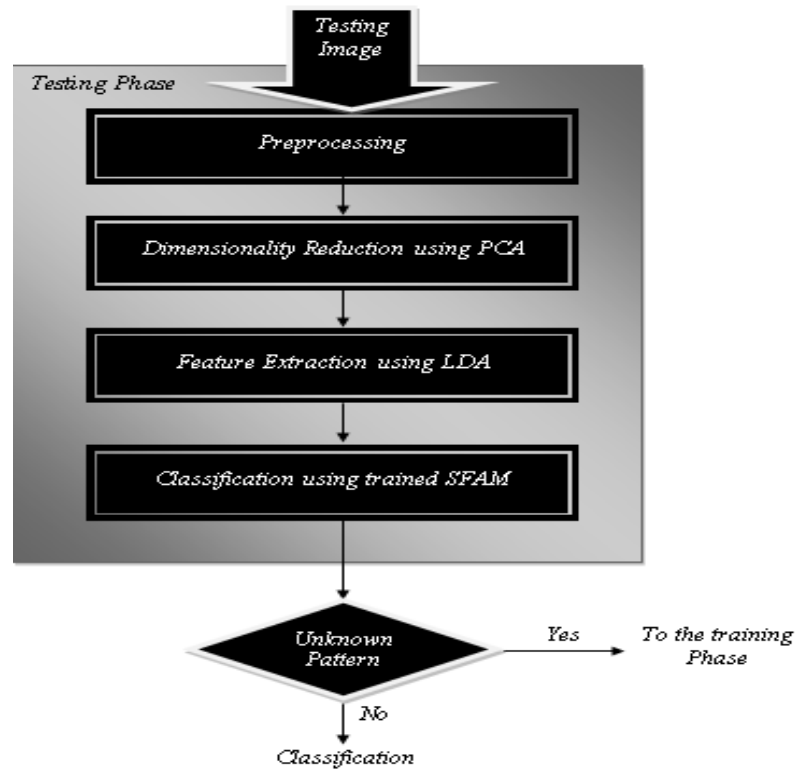

Figure 4(b). Testing Phase of the Proposed Face Recognition System

The system consists of four steps: Preprocessing, Dimensionality reduction using PCA, Feature extraction using LDA and Classification using SFAM.

The detailed explanation and implementation details of each step are given in the next session.

\section{IMPLEMENTATION}

Face recognition method is applied on ORL face dataset for separation of twenty classes. Out of 10 face images of each person 7 are used for training the network. Thus when we consider 20 classes there are 140 training face images. Figure 5 shows examples of sample images used.

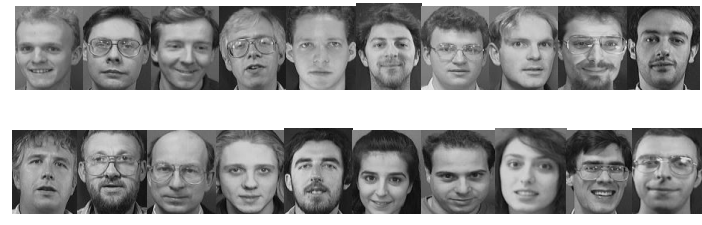

Figure 5. Examples of Sample Face images used

Figure 6 gives the diagrammatic representation of step by step process followed in the proposed system. 


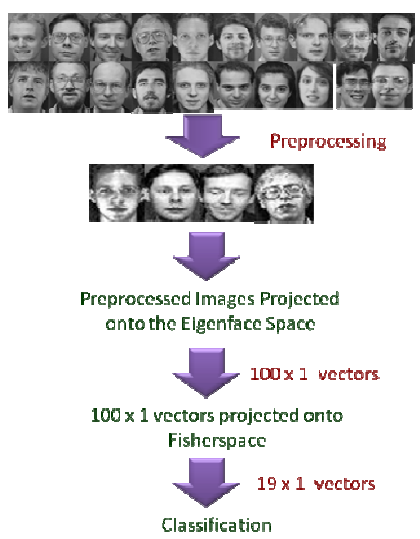

Figure 6. Steps of Implementation

Detailed explanation of each step in the system is given below.

\subsection{Preprocessing}

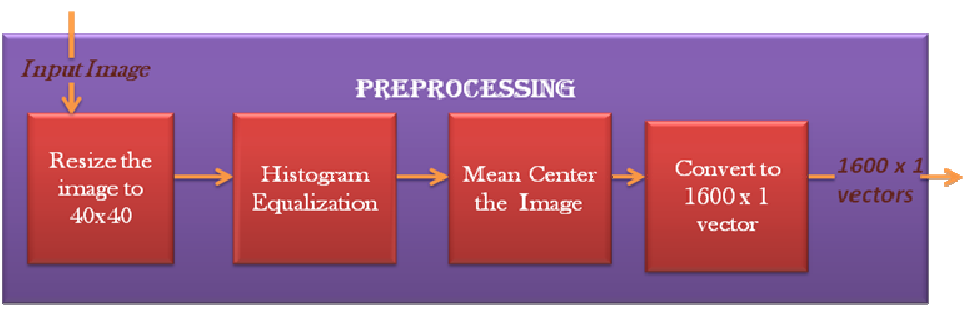

Figure 7. Flowchart of Preprocessing Step

In this phase first we resize the images to $40 * 40$ sizes. After that we histogram equalize all input images in order to spread energy of all pixels inside the image. As a next step, we subtract mean images from face images to mean center all of them. Finally all preprocessed face images change to a vector $(1600 \times 1$ vector $)$ and go to the next step. Figure 8 shows example of preprocessed images and Figure 7 shows the flowchart of preprocessing step.

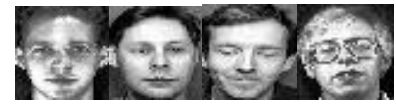

Figure 8. Examples of preprocessed images

\subsection{Dimensionality Reduction using PCA}

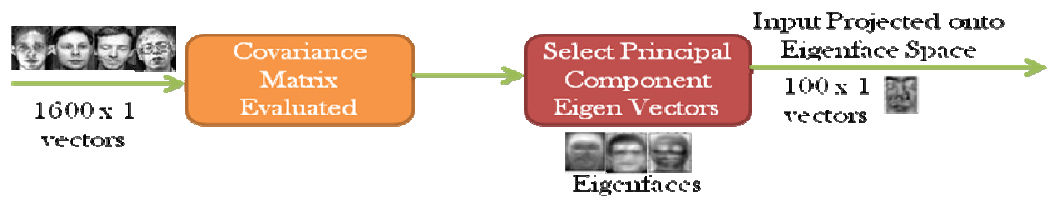

Figure 9. Flowchart for Dimensionality Reduction step

Figure 9 gives the flowchart for Dimensionality Reduction step. Every input image is cropped to $40 \times 40$ image in the preprocessing step; as a result the input of this stage is a preprocessed $1600 \times 1$ vector. These vectors are used to estimate the covariance matrix. After estimation of the covariance matrix, significant eigenvectors of the covariance matrix are computed. Number of 
Signal \& Image Processing : An International Journal(SIPIJ) Vol.1, No.2, December 2010

eigen-vector depends on the accuracy that the application demands. Large number of eigenvectors will obviously improve the accuracy of the method but computational complexity will increase in this step and next step. Thus considering accuracy and computational complexity 100 eigen vectors are selected as principal eigen vectors.

The preprocessed faces are now projected on to the space with 100 eigenfaces as base vectors to get $140,100 \times 1$ vectors. Thus dimensionality reduction phase converts $1600 \times 1$ vectors to $100 \times 1$ vectors.

\subsection{Feature Extraction using LDA}

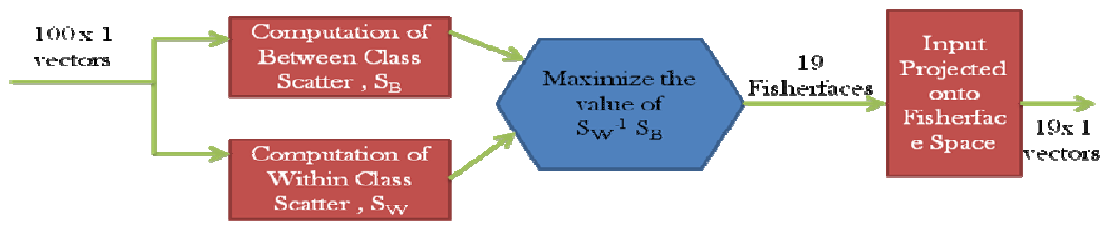

Figure 10. Flowchart for Feature Extraction using LDA

Figure 10 shows the flowchart of Feature Extraction step. Outputs of dimension reduction part are $100 \times 1$ vectors. Features are extracted from these vectors. 19 principal components which are selected from among the eigen vectors form the features. The preprocessed face images projected on to the eigen space, is now, projected onto the fisher space and thus results in 140, $19 \times 1$ vectors which are used as input of neural network.

\subsection{Classification using SFAM}

For the direct application of SFAM to the face recognition problem, the architecture is trained using the $140,19 \times 1$ vectors got from the feature extraction stage. The vigilance parameter is selected so that the number of categories to which the network settles is same as the number of classes in the training data.

The network took only 2 epochs for completing its learning or training phase. The time that it took for learning is negligible (order of seconds) and thus can be labeled as very efficient in terms of time complexity.

Once the training is over and the top-down weight matrices have been obtained, the testing can be carried out using the matrix equivalent of the test input image.

The most important point to note about the network in the classification stage is that it remains open to adaptation in the event of new information being applied to the network. If an unknown pattern is applied to the network SFAM will always attempt to assign a new class in the category output layer by assigning the unknown input to a node.

Classification using BPNN was also implemented for comparison purpose. Figure 11 shows the architecture of BPNN used for classification. The network implemented consists of an input layer with 19 neurons, two hidden layers first with 30 and second with 25 neurons respectively and an output layer with 10 neurons. A simple back propagation algorithm is used to train the network.

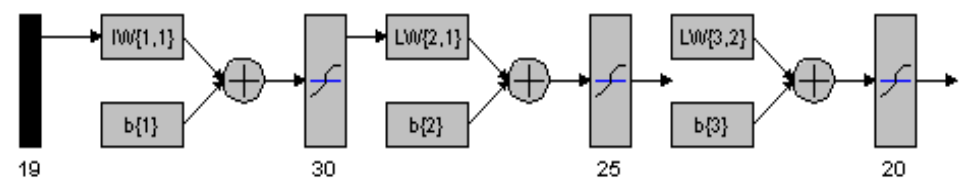

Figure 11. Architecture of BPNN implemented 


\section{RESULTS}

Recognition rate was observed by varying number of training images and testing images. Experiment was conducted on ORL Database.

\subsection{SFAM proved to be a speedy and accurate recognizer}

Table 1. Result of the Experiment Conducted on ORL Database

\begin{tabular}{|c|c|c|c|c|c|}
\hline $\begin{array}{c}\text { No: of Training } \\
\text { Images Used for } \\
\text { Each Subject }\end{array}$ & $\begin{array}{c}\text { No: of } \\
\text { Testing } \\
\text { Images } \\
\text { Used for } \\
\text { Each } \\
\text { Subject }\end{array}$ & $\begin{array}{c}\text { Name of the } \\
\text { Classifier }\end{array}$ & No :of Epochs & $\begin{array}{c}\text { Elapsed Time } \\
\text { (in seconds) }\end{array}$ & $\begin{array}{c}\text { Recognition } \\
\text { rate }\end{array}$ \\
\hline \multirow{2}{*}{7} & 3 & BPNN & 100 & 1255.03 & $95.5 \%$ \\
\cline { 3 - 6 } & \multirow{2}{*}{6} & SFAM & 2 & 0.125 & $98.5 \%$ \\
\cline { 3 - 6 } & \multirow{2}{*}{5} & BPNN & 100 & 1250 & $94 \%$ \\
\hline \multirow{2}{*}{5} & 5 & SFAM & 2 & 0.115 & $98 \%$ \\
\cline { 3 - 6 } & & SPNN & 100 & 1253 & $93 \%$ \\
\hline
\end{tabular}

Table 1 shows the result of the test conducted on ORL database. Three cases (number of training samples taken as 7,6 \& 5) are considered here.

The recognition rate for system using SFAM as classifier is found to be higher than the recognition rate for system using BPNN.

The training time for SFAM is negligible when compared to BPNN. The training time for BPNN is about 10,000 times that of training time for SFAM. This adds to the advantage of the system using SFAM as the classifier. 
Signal \& Image Processing : An International Journal(SIPIJ) Vol.1, No.2, December 2010

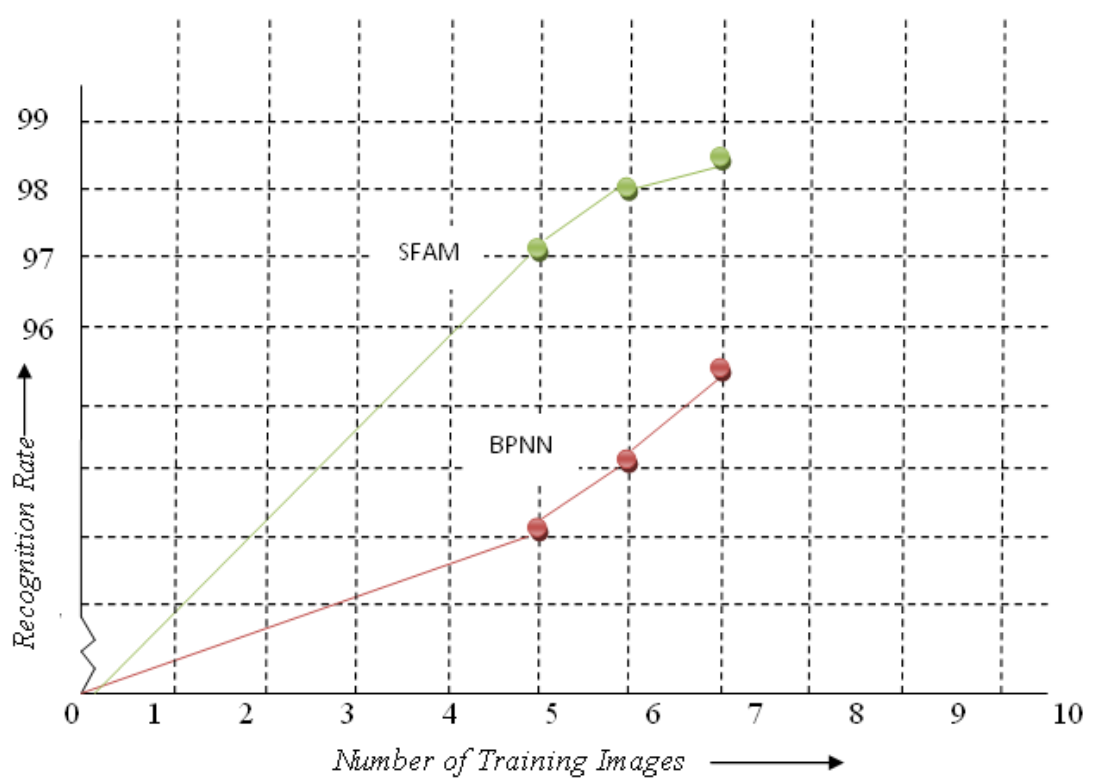

Figure 12. Graph showing the Result of experiment conducted on ORL Database

Figure 12 shows the plot of recognition rate versus number of training samples, when the test is carried out on ORL Database. The graph shows that the recognition rate for the system using SFAM as the classifier is higher when compared to the system using BPNN as the classifier.

The above result analysis show that SFAM exhibits a very good performance both in terms of recognition rate and time required to train the net. The recognition rate can be improved by increasing the number of eigen vectors selected as Principal Components in the dimensionality reduction step.

The network took only 2 epochs for completing its learning or training phase. The time that it took for learning is negligible (order of seconds) and thus can be labeled as very efficient in terms of computer time. Thus SFAM proves itself to be a classifier that learns very fast when compared to Back Propagation Neural Network that takes more than 1200 seconds to converge when trained for the same input.

Thus recognition system using SFAM can perform as an efficient recognizer in cases where a speedy and accurate system is required.

\subsection{SFAM proved to be adaptive}

When the face recognition system using BPNN as the classifier is given any input image that belongs to a class for which system is not trained for, it will converge to the conclusion that it belongs to one of the class for which it is trained.

An example is shown in Figure 13. Figure 13(a) shows the test input belonging to the class for which the system is not trained for. 


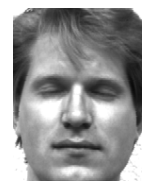

Figure 13(a) Test Image belonging to the class, network is not trained for

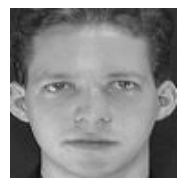

Figure 13(b) Classification that BPNN yields

Figure 13(b) shows the class to which the system using BPNN as the classifier converges.

But in the case of SFAM it conclude that the input does not belong to any class for which it trained for by giving the output as classification $=-1$, then the network learns this input as a pattern belonging to a new class.

After this learning procedure if the network come across this input again it classifies it to the new class it created.

Thus from this result it is obvious that the system using SFAM as the classifier is open to adaptation in the event of new information being applied to the network. If the system using SFAM as the classifier encounters a face image that it is not trained for, it will conclude that it is a face that it is seeing for the first time and it learns the new pattern. But in the case of system using BPNN as the classifier erroneous input (test input not belonging to any of the class for which it is trained for) yields erroneous result.

Since SFAM can adaptively be trained, this can be employed in the cases, like student record maintenance at institutions, attendance keeping at organizations and so on, where addition to database needs to be made frequently.

\section{CONCLuSiON}

In this work a new, SFAM based Face recognition, method is presented. Proposed method consists of four parts: i) Image Preprocessing that includes histogram equalization, and mean centering, ii) Dimensionality reduction using PCA where main features that are important for representing face images are extracted iii) Feature Extraction using LDA where significant features for class separability are selected and iv) SFAM classifier classifies input face images into one of available classes if the test input belongs to a class for which it is trained for and if not the system concludes that it is an input belonging to a class it is not trained for and will learn this new pattern. Back Propagation Neural Network is also implemented in this work for the comparison purpose. When compared to BPNN, SFAM acts as a more powerful classifier by exhibiting negligible computer time for training and a solution to Stability Plasticity Dilemma. Simulation results using ORL, YALE and Indian Face Database demonstrated the ability of SFAM over BPNN for efficient face classification. Not only did SFAM made the face recognition system adaptive and produced a high recognition rate but also reduced the training time by around 10,000 times when compared to BPNN. Thus this work proves system using SFAM as the classifier to be a speedy (negligible training time), secure ( high recognition rate) and adaptive face recognition system. Future works include enriching the system to attain more human-like recognition capabilities and consequently provide tools that are more targeted to user's actual needs. This target in the future could be met by making the system successful even in vastly varying pose and lighting conditions. This is possible only when feature extractors are extracting features such that this type of improved recognition is possible. So powerful feature extractors can be employed to extract features strong enough to make the recognition possible in much less constrained situations. This work has proved SFAM (as a classifier) to be successful both in terms of recognition rate and training time required, for the given set of features, when 
Signal \& Image Processing : An International Journal(SIPIJ) Vol.1, No.2, December 2010

compared to other classifiers. So the features extracted with more powerful feature extractors along with SFAM as the classifier will turn out the system to be more human like.

\section{REFERENCES}

1. Jain, R. Bolle, S. Pankanti Eds, "BIOMETRIC - Personal Identification in Networked Society", Kluwer Academic Publishers, Boston/ Dordrecht/ London, 1999.

2. J. R. Solar, P. Navarreto, " Eigen space-based face recognition: a comparative study of different approaches, IEEE Tran. , Systems man And Cybernetics- part c: Applications, Vol. 35, No. 3, 2005.

3. Sahoolizadeh, H.; Ghassabeh, Y.A.;" Face recognition using eigen-faces, fisher-faces and neural networks", Cybernetic Intelligent Systems,2008. CIS 2008.7th IEEE International Conference on 9-10 Sept. 2008,Page(s):1 - 6 Digital Object Identifier 10.1109 / UKRICIS. 2008.4798953

4. M. Turk, A. Pentland, "Eigen faces for face recognition", Journal cognitive neuroscience, Vol. 3, No.1, 1991

5. W. Zhao, R. Chellappa, A, Krishnaswamy, "Discriminant analysis of principal component for face recognition", .IEEE Trans. Pattern Anal. Machine Intel., Vol 8, 1997.

6. O.Deniz, M. Castrill_on, M. Hern_andez, "Face recognition using independent component analysis and support vector machines", Pattern Recognition letters, Vol. 24, PP. 2153-2157, 2003.

7. John Wright, Allen Y.Yang, Arvind Ganesh, S. Shankar Sastry and Yi Ma, "Robust Face Recognition via Sparse Representation," IEEE Trans. On Pattern Analysis and Machine Intelligence, vol. 31, no. 2, pp. 210-225, 2008

8. Thang V.Pham and Arnold W.M. Smeulders, "Sparse Representation for Fine and Coarse Object Recognition," IEEE Transactions on Pattern Analysis and Machine Intelligence, vol. 28, no. 4, pp. 555-567,2006.

9. K. Lee, Y. Chung, H. Byun, "SVM based face verification with feature set of small size", electronic letters, Vol. 38, No. 15, PP. 787-789, 2002.

10. H. Othman, T. Aboulnasr, " A separable low complexity 2D HMM with application to face recognition" IEEE Trans. Pattern. Anal. Machie Inell., Vol. 25, No. 10, PP. 1229-1238, 2003.

11. M. Er, S. Wu, J. Lu, L.H.Toh, "face recognition with radial basis function(RBF) neural networks", IEEE Trans. Neural Networks, Vol. 13, No. 3, pp. 697-710.

12. M.J.Er, W.Chen, S.Wu, "High speed face recognition based on discrete cosine transform and RBF neural network", IEEE Trans. On Neural Network, Vol. 16, No. 3, PP. 679,691, 2005.

13. Z. Pan, A. G. Rust, H. Bolouri,, " Image redundancy reduction for neural network classification using discrete cosine transform”, Proc. Int. Conf. on Neural Network, Vol. 3, PP. 149,154, Italy, 2000.

14. [14] Nazeer, Shahrin Azuan, Nazaruddin Omar, and Marzuki Khalid. "Face Recognition System using Artificial Neural Networks Approach." International Conference on Signal Processing, Communications and Networking. Chennai, India: IEEE, 2007. 420-425

15. Ming Gu Jing-Zhou Zhou Jian-Zhong Li "Online face recognition algorithm based on fuzzy ART" ,International Conference on Machine Learning and Cybernetics, 12-15 July 2008, Vol. 1, Page(s): 556 - 560

16. M.A. Turk, A.P. Pentland, "Face Recognition Using Eigenfaces", Proceedings of the IEEE Conference on Computer Vision and Pattern Recognition, 3-6 June 1991, Maui, Hawaii, USA, pp. 586-591

17. D.L. Swets and J.J. Weng, "Using Discriminant Eigen features for image retrieval", IEEE Trans. Pattern Anal. Machine Intel, vol. 18, PP. 831-836, Aug. 1996.

18. A. M. Martinez, A. C. Kak, "PCA versus LDA", IEEE Trans. Pattern Anal. Machine Intell, Vol. 23, pp. 228-233. 2004. 
Signal \& Image Processing : An International Journal(SIPIJ) Vol.1, No.2, December 2010

19. P.N. Belhumeur, J.P. Hespanha, and D. J. Kriegman, "Eigen faces vs. Fisher faces: Recognition using class specific linear projection", IEEE Trans. Pattern Anal. Machine Intel., vol. 19, PP. 711-720, may 1997.

20. Papoulis, A. \& Pillai, U. (2002)."Probability, random variables, and Stochastic Processes", McGraw-Hill, 0073660116, New York

21. Haykin, S. (1999) "Neural Networks: A comprehensive foundation, Prentice Hal"l, 0-13273350-1,New Jersey

22. Eleyan, A. \& Demirel, H. (2006). "PCA and LDA Based Face Recognition Using Feedforward Neural Network Classifier", Proceedings of Multimedia Content Representation,Classification and Security, pp. 199-206, 978-3-540-39392-4, Turkey, September 2006,Springer-Verlag, Istanbul

23. S.Rajasekaran and, G.A.Vijayalakshmi Pai," Image Recognition Using Simplified Fuzzy ARTMAP Augmented with a Moment based Feature Extractor", International Journal of Pattern Recognition and Artificial Intelligence, Vol. 14, No. 8 (2000) 1081-1095

24. S.Rajasekaran and, G.A.Vijayalakshmi Pai, "Neural Networks, Fuzzy Logic, and Genetic Algorithms", Prentice-Hall of India, New Delhi, 2003.

25. T. Kasuba, "Simplified fuzzy ARTMAP," AI Expert, November (1993) 18-25.

26. S. Rajasekaran and V. Pai, "Simplified fuzzy ARTMAP as a pattern recognizer", J. Comput. Civil Engin. ASCE 14, 2 (2000) 92-99.

27. Kwang In Kim, Keechul Jung, and Hang Joon Kim, "Face Recognition Using Kernel Principal Component Analysis", IEEE Signal Processing Letters, February 2002, Vol. 9, No:2, 40-42

28. R.Beale and T.Jackson,” Neural Computing”,Institute of Physics publishing, Bristol and Philadelphia, 2001. 